NASATECMNDCAL

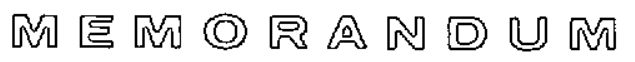

NASA TM X-71703

栗

by R. G. Deissler

Lewis Research Center

Cleveland, Ohio 44135

TECHNICAL PAPER to be presented at

Fundamentals and Applications of Turbulence Lecture University of Tennessee Space Institute

Tullahoma, Tennessee, April 21-25, 1975

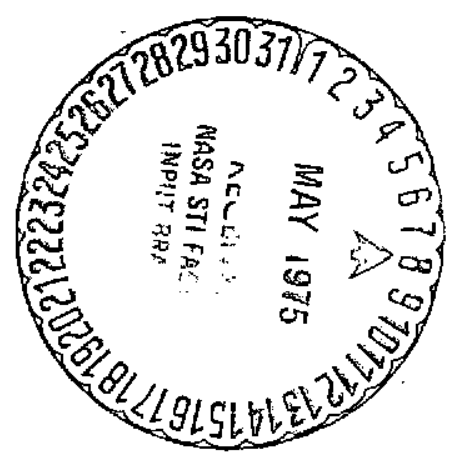




\title{
TURBULENCE PROCESSES AND SIMPLE CLOSURE SCHEMES
}

\author{
R. G. Deissler \\ Lewis Research Center \\ National Aeronautics and Space Administration \\ Cleveland, Ohio 44135
}

The closure problem in turbulence is briefly reviewed, and some simple closure schemes are introduced. Processes occurring in turbulent flow are discussed on the basis of solutions for some elementary flows such as homogeneous turbulence with and without uniform shear. Closure by specification of initial conditions, and finally practical closure schemes for more complicated flows (e.g., pipe flows) are considered briefly. The latter include Reynolds stress, eddy viscosity, and mixinglength closures.

The origin of the closure problem in turbulence was discussed in some of the earlier papers in this volume. Herein we briefly review the closure problem and introduce simple closure schemes in order to obtain solutions for some simple flows. These solutions will be used to illustrate the processes occurring in turbulence. Closure by specification of initial conditions will then be considered. Finally, practical closure schemes for more complicated flows, such as boundary layers and pipe flows will be discussed.

In order to illustrate the important processes of turbulence dissipation and turbulence transfer between wave numbers or eddy sizes we first consider a statistically homogeneous turbulent field without mean gradients. Such a turbulence will decay with time since no energy is added to the system, and so we must consider an initial value problem. The turbulence must be generated initially by some means, as by flow through a grid.

We start the analysis by writing the Navier-Stokes equations at two arbitrary points $P_{\rightarrow}$ and $P^{\prime}$ in the turbulent fluid, separated by the vector $\vec{r}$. (See Fig. 1 , where the subscripts in the equations can take on the values 1,2 , or 3 , 


\section{R. G. Deissler}

and a repeated subscript in a term indicates a sumation. The quantities $u_{i}$ and $u_{j}$ are instantaneous velocity components, $x_{i}$ is a space coordinate, $t$ is the time, $\rho$ is the density, $v$ is the kinematic viscosity and $p$ is the instantaneous pressure.) Then we multiply the equation at point $P$ through by a velocity component at $P^{\prime}$, and that at $P^{\prime}$ by a velocity component at $P$, add the equations, take average values and finally arrive at the equation involving correlations between quantities at points $P$ and $P^{P}$ shown at the bottom of Figure 1.1 Besides velocity-velocity correlations, the equation contains pressure-velocity correlations and triple-velocity correlations. The equation can be converted to spectral form by taking its three-dimensional Fourler transform and setting $i=j$, as shown at the top of Figure 2, where $k$ is the wave number, $E$ is the energy spectrum function, and $W$ is the energy transfer term due to triple correlations.1 Note that this operation also converts the partial differential equation to an ordinary one. The various terms in the spectral equation can be interpreted by multiplying the equation through by a wave-number band of width $\mathrm{dk}$ and referring to the sketch in Figure 2. The energy spectrum function $E$ gives contributions from various eddy sizes to the total turbulent energy. The area under the spectrum curve is thus equal. to the total turbulent energy $\overline{u_{i} u_{i}} / 2$. The first term in the spectral equation represents the rate of change of turbulent energy in the crosshatched wave-number band. The next term $W d K$ represents the net rate of transfer of energy into the wave-number band by nonlinear effects; that is by the triple correlation term in Figure 1. Finally, the last term $2 \nu K^{2} \mathrm{E} d K$ represents the rate at which turbulent energy is dissipated within the wave number band by viscous action. The dissipation term is always negative, in contrast to the transfer term. Note that a spectral equivalent of the pressure-velocity correlations is absent in this equation for $E$ dK the total turbulent energy in a wave number band. This indicates that the pressure-velocity correlations do not contribute to the rate of change of the turbulent energy but they can transfer energy between its directional components.

Unfortunately the spectral equation contains two unknowns, $E$ and $W$, so that we cannot in general obtain a solution without more information. That is, of course, a manifestation of the closure problem in turbulence, and is a consequence of the nonlinearity of the original Navier-Stokes equations. However, if the turbulence is sufficiently weak, the inertia or transfer term $W$ will be negligible, and we can obtain, subject to particular initial conditions, the remarkably simple solution shown at the bottom of Figure $2.1 \mathrm{~J}_{0}$ and $t_{0}$ are constants 
that depend on initial condftions. Although this solution applies only to very low Reynolds numbers, it can be used to study the viscous dissipation, the only turbulence process accounted for in its derivation. If we set $\partial \mathrm{E} / \partial \kappa=0$, we get $1 / \sqrt{\nu\left(t-t_{0}\right)}$ for $k_{m}$, the wave number at which $E$ is a maximum. Thus as time increases, the turbulent energy shifts to lower wave numbers, or to larger eddy sizes. The physical interpretation of this shift is that the smaller eddies die out faster than the larger ones because of the larger velocity gradients (larger shear stresses) between the smaller eddies. The essence of the turbulence dissipation process appears to be that the dissipation is always negative and that it affects malnly the smaller eddies.

Unless the turbulence level is very low, as in the final period of decay, the inertia or transfer effects will not be negligible ( $W$ \& 0 ), and so we would like to be able to take them into account in some way. A large number of proposals for calculating the transfer function $W$ have been given including those of Heisenberg, 2 Kovasznay, 3 and Kraichnan.4 There are in fact almost as many proposals for the transfer function as there are workers in turbulence. For our purposes it will be sufficient to consider a simple deductive approach which is essentially a perturbation on the solution for the final period of decay. 5,6

In carrying out the analysis we consider in addition to the two-point correlations of Figure 1, three, and sometimes four or more points. Thus, we can write the Navier-Stokes equations at three and at four arbitrary points in the turbulent fluid, as in Figure 3. We can then construct three-point correlation equations involving triple and quadruple correlations, and four-point equations involving quadruple and quintuple correlations, as shown in the figure. However, there are still more unknowns than there are equations, again as a result of the nonlinearity of the Navier-Stokes equations. To make the system of equations determinate, we use an operation similar to that used for the final period of decay, but instead of assuming that the turbulence is weak enough to neglect the inertia term in the two-point equation, we assume only that it is weak enough to neglect the inertia term in the highest-order equation considered. Herein, in order to give the simplest posstble representation of the transfer or inertia process, we use only two- and three-point equations, 5 although the analysis has also been carried out for four points. ${ }^{6}$ Thus, neglecting the quadruple correlations in the three-point equation gives a determinate set of equations that should be applicable at times somewhat before the final period. 


\section{R. G. Deissler}

The resulting energy transfer spectra are plotted in Figure 4.5 They are negative at small wave numbers and positive at higher ones, indicating that energy is transferred out of the low wave-number region and into the higher wave-number region. The net areas under the curves are zero, since the term makes no contribution to the rate of change of the total turbulent energy. The transfer of energy to the high wave number or small eddy region can be thought of as a breakup of big eddies into smaller ones, or alternatively as a stretching of vortex filaments (to smaller diameters).

Calculated energy spectra are plotted for various times in Figure 5.5 The curves show how the spectrum changes shape with time and approaches that for the final period. For small times the inertia or transfer terms transfer energy into the high wave number or small eddy region and cause the slopes on the high wave number sides of the spectra to be less steep than they are at larger times, when the spectrum assumes a more or less symmetrical shape. Thus, the function of the inertia terms in the equations is to excite the higher wave number or sma11 eddy regions of the spectrum by transferring energy into those regions. If it were not for inertia effects, those regions of the spectra would be absent, as they are in the final period of decay.

Inertia and dissipation tend to shift the energy in opposite directions on the wave-number scale. However, the mechanisms for the two effects appear to be different. Whereas inertia tends to transfer the energy to higher wave numbers by a breakup of large eddies into smaller ones (or by a stretching of vortex filaments), dissipation tends to shift the energy to smaller wave numbers by selective annihilation of eddies, the small eddies being the first to go. As the turbulence decays, the dissipation effects must, of course, eventually win out, since the inertia effects become negligible at the low Reynolds numbers occurring at large times.

Before going on to the effects of mean gradients on turbulence, I should like to briefly consider closure by specification of initial conditions. In the closure methods just considered, we note that as we use more points in the turbulent field, or higher-order correlation equations, we have to specify more initial conditions to solve the set of equations (since there are more dependent variables). This is in addition to the closure assumption which must be made for the highest order correlation. If we are willing to specify multiple initial conditions, as indeed we must for a complete specification of the initial turbulence, 1 there is an alternative way of looking at the problem which does not require an assumption for 


\section{Turbulence Processes and Simple Closures}

the highest order correlation. It is not hard to show that, given the initial multipoint correlations and their correlation equations, all of the time derivatives of the turbulent energy tensor and of other pertinent turbulence quantities can be calculated. 7 These time derivatives can then be used in a series to calculate the evoluation of the turbulent energy tensor (or of the equivalent energy spectrum tensor) and of other turbulence quantities.

When the turbulence is treated in this way, we no Ionger have the usual problem of closing an infinite set of correlation or spectral equations. The correlation equations are used only to relate the correlations at an Initial time to their derivatives, and those correlations must be given in order to have a complete specification of the turbulence at that time. of course, in practice only a small number of the correlations, and thus of their time derivatives will ordinarily be available but a sufficient number may be known to give a reasonably good representation. In the theory of Ref. 7 it was found that by specifying $n$ spectra at an inttial time, where $n$ is an odd integer greater than or equal to 3 , the evolution in time of those $\mathrm{n}$ spectra was predictable. Good agreement with experimental data was obtained for $n=3$ or $5.9,8$

It may be that the nature of the problem is such that three or more spectra have to be specified initially in order to calculate the evolution of any of them (except for weak tur bulence). However, particularly in an applied problem, three or more initial spectra will of ten not be available. In that case possible courses of action are first, the required initial spectra might be estimated from previous experimental or analytical results or second, the introduction of physical or mathematical hypotheses into the theory might be allowed. In Ref. 9 the latter course of action is followed by assuning that the energy transfer is a function of the initial conditions and of the energy at $k$. By allowing that hypothesis it is found that by specifying only two initial spectra, $E$ and $W$, the evolutions of those spectra are predictable. The results are compared with experiments in Figures 6 to 8 , where $A$ is a constant for the inttial conditions. As in the theory of Ref. 7 the present theory 9 contains no adjustable constants or functions.

We turn now to the important case of turbulent shear flow. In order to consider a solvable problem in which we can study the turbulence processes associated with the mean shear, we use for our model a homogeneous turbulent field with a uniform mean shearing velocity gradient. Since we have already considered the energy transfer process, it is assumed in this case that 


\section{R。 G. Delssler}

the interaction between the turbulence and the mean gradient is large compared with the turbulence self-interaction. Thus, we need only consider the two-point correlation equations, since we can close them if we neglect the self-interaction or triple correlation terms by comparison with the mean gradient terms. The analysis is similar to that just described, except that we break the velocity into mean and fluctuating components. In that way we obtain equations for the $\overline{u_{i} u_{j}}$ and their Fourier transforms in the presence of a uniform velocity gradient. 10

The resulting equation for the energy spectrum is

$$
\frac{\partial E}{\partial t}=P \frac{\partial U_{1}}{\partial x_{2}}+T \frac{\partial U_{1}}{\partial x_{2}}-2 \nu \kappa^{2} E
$$

As in the equation at the top of Figure 2, the first term gives the rate of change of the energy spectrum function $E$ at wave number $k$. The next term is proportional to the mean shearing velocity gradient, and $P(\kappa)$ in that term is proportional to a spectral component of the turbulent shear stress. That term is thus interpreted as a production term by which energy is produced at wave number $\kappa$ by work done by the velocity gradient on a spectral component of the turbulent shear stress.

The third term is also proportional to the mean velocity gradient, but we interpret it as a transfer term rather than as a production term. That is because if we integrate the term over $k$ from 0 to $\infty$, we get the result 0.10 The term gives zero contribution to the rate of change of the total turbulent energy, but it can transfer energy between wave numbers. Thus, a1though we neglect triple correlations, we still get an energy transfer $T$ which appears simflar to that produced by triple correlations. The difference between the two cases is that, whereas in the case of triple correlations, the transfer is produced by the nonlinear action of the turbulence on itself, in the present case it is due to the external action of the mean velocity gradient on the turbulence. However, the results in both cases are similar, as shown in Figure 9 for a particular initial condition. 10 Since the dimensionless transfer term is primarily negative at low wave numbers and positive at higher ones (net area $=0$ ), the energy transfer is mostly from small to large wave'numbers, as is the case for energy transfer by triple correlations. The reason the dimensionless $T^{*}$ can increase with time is that $\mathrm{T}^{*}$ itself contains time.10

Finally, the last term in the spectral equation is again the dissipation term. 
Plots of the energy spectrum, production term, and dissipation term for a particular time are shown (normalized to the same height) in Figure 10.10 From these we can sumarize the history of the turbulent energy as follows. The energy comes into the turbulent field from the mean velocity field mainly through the large eddies. This energy is then transferred from the big eddy region to the small eddy region by the transfer term just discussed. Physically this transfer might be thought of as being produced by the stretching of turbulent vortex filaments by the mean velocity gradient. Finally the energy is dissipated in the small eddy region by viscous action. It is physically reasonable that the dissipation should occur mainly in the sma1l eddy region since the shear stress should be greater between the small eddies than between the larger ones. The energy resides in a region between the production and dissipation regions, as shown by the plot of the energy spectrum.

By using realistic initial conditions the analysis has been compared with experiments for uniformly sheared turbulence in Ref. 11. Some of the results are shown in Figures 11 and 12 . The agreement is good, including a negative region in one of the two-point correlation curves.

Although the agreement between theory and experiment is good for the data shown in Figures 11 and 12, it should be pointed out that the results are not directly applicable to fully developed inhomogeneous flows (e.g., pipe flows), since all of the predicted components of the turbulence decay at large times. This is shown by the dashed curves in Figure 13.12 The decay apparently occurs because there are no production terms in the full equations for $u_{2}^{2}$ and $u_{3}^{2}$, the transverse turbulence components. In order to test that hypothesis, $\mathrm{u}_{2}^{2}$ and $\overline{u_{3}^{2}}$ were arbitrarily set equal to $(1 / 2) \mathrm{u}_{1}^{2}$. The solid curves in Figure 14 indicate that when this is done, all of the components grow at large times.

Thus, for simulating a sustained turbulence it is important that the energy remain distributed among the various directional components of the turbulence. One way of maintaining this directional distribution (other than by forcing it as in Fig. 13) is by stretching the turbulence in the direction of flow as it is being sheared $\left(\partial U_{1} / \partial x_{1}>0\right)$. This action tends to put energy into the transverse components, so that the turbulence is malntained at large times. This is shown by the solid curves in Figure 14,12 where all of the turbulence components grow at large times. 
In a fully developed pipe flow, where longitudinal straining is absent, the distribution of the turbulent energy between its directional components might be maintained by an interaction of triple correlations with the pressure-velocity correlations. That effect was neglected in the analysis considered here, although the effect of mean gradients on the pressurevelocfty: correlations was included. Inhomogeneities in the turbulent field may also have a directional redistribution effect on the turbulent energy. 13

This discussion leads us to a consideration of more complicated turbulent flows than those considered thus far, such as those in boundary layers and pipes. A fully developed turbulent pipe flow may seem simple, but in reality it is exceeding1y complicated when approached from a fundamental standpoint. A complete solution for an inhomogeneous turbulent shear flow has not been obtained, although some progress has been made in Ref. 13. Work to obtain a more complete solution is currently in progress, but it is too early to say whether it will be successful.

The complete two-point correlation equations for the double velocity and pressure-velocity correlations for an inhomogeneous flow with mean velocity gradients are given in Ref. 10. The principa1 process occurring in inhomogeneous turbulence which has not been considered thus far is turbulence diffusion between regions of higher and lower turbulence intensity. We have not been able to obtain solutions to illustrate the diffusion process, except for viscous diffusion. 10 Since the two-point equations for inhomogeneous turbulence are extremely complex, we content ourselves here with considering only the one-point equations, the equations for the mean flow. Those equations are $\mathrm{e}^{10}$

$$
\frac{\partial U_{i}}{\partial t}+U_{k} \frac{\partial U_{i}}{\partial x_{k}}=-\frac{1}{\rho} \frac{\partial p}{\partial x_{i}}+\frac{\partial}{\partial x_{k}}\left(v \frac{\partial U_{1}}{\partial x_{k}}-\overline{u_{i} u_{k}}\right)
$$

where the overbars and the $U_{i}$ designate mean values. These equations were obtained from the Navier-Stokes equations by breaking the instantaneous velocities and pressures into mean and fluctuating components. The system of equations is closed in this case by Introducing assumptions for the $\overline{u_{i} u_{j}}$.

It is an interesting observation that as we have gone from comparatively simple to more complex systems we have been forced to consider less points in the turbulent fluid, and to use lower-order closures. Thus for homogeneous turbulence without mean gradients we were able to obtain a solution by 
closing the three- or four-point correlation equations. When uniform mean gradients were added, closure was obtained at the two-point level. Finally for inhomogeneous turbulence we will consider only the one-point equation (Eq. (1)). Many workers have also used higher-order one-point equations, which are somewhat easier to solve than the higher-order multipoint equations. Some of those methods will be considered in later papers in this volume. However, it should be pointed out that when higher-order one-point equations are introduced, the number of unknowns goes up faster than the number of equations, so that the amount of empirical information which must be supplied is greatly increased. Nevertheless, such equations have been found useful in some cases.

If we restrict our attention to flows for which the boundary-1ayer assumptions are applicable, the only component $\overline{u_{1} u_{k}}$ in the Equation (1) which we need consider is $\overline{u_{1} u_{2}}$, the Reynolds shear stress, where the shear velocity gradient is $\partial U_{1} / \partial x_{2}$. The above system of equations then reduces to a single equation. One flow for which that equation can be easily closed is that in a moderately short boundary layer with a severe pressure gradient. It is shown in Ref. 14 that for this case $\overline{u_{1} u_{2}}$ can be considered as frozen at its initial given values as it is convected along stream lines. Theoretical velocity profiles and Stanton numbers (dimensionless heat transfer coefficients) are compared with experiment in Figures 15 and $16.14,15$ The quantity $\tau_{w}$ is the shear stress at the wall. For obtaining the heat transfer results, an energy equation simflar to the above equation for the mean flow (Eq. (1)) was used in addition to that equation. The agreement between theory and experiment is considered good.

The above analysis is an example of a case in which closure can be obtalned in a simple manner at the Reynolds stress leve1. For longer boundary layers, or for less severe pressure gradients, the simplification used breaks down, and so other less deductive methods have to be used. The reason a solution could be obtained is that for the case considered, the given initial conditions were all-important. At the other extreme we have fully-developed flows, where initial conditions have no effect at all on the turbulence. For that case and for other, intermediate, cases; we generally employ simple models based on physical reasoning and dimensional analysis. As mentioned above, if we make the boundary-layer assumptions, we need consider only the $\overline{u_{1} u_{2}}$ component of the Reynolds stress $\overline{u_{i} u_{j}}$ in the one-point Equation (1).

There are at least three types of closure schemes we can use. First, we can make an assumption for $\overline{\mathrm{u}_{1} \mathrm{u}_{2}}$ directly 
(Reynolds shear-stress closure). Second, by analogy with the molecular viscosity, we can introduce an eddy viscosity $\varepsilon$ given by

$$
\overline{u_{1} u_{2}} \equiv-\varepsilon \quad \partial U_{1} / \partial x_{2}
$$

and make an assumption for $\varepsilon$. Finally, we can introduce a mixing length for the turbulence (Prandtl's mixing length ${ }^{16}$ ) given by

$$
\overline{u_{1} u_{2}} \equiv-\ell^{2}\left(\partial u_{1} / \partial x_{2}\right)^{2}
$$

and make an assumption for $\ell$. Which of these three closure schemes is used is to some extent a matter of taste although one will sometimes be more convenient than another. The eddy viscosity or the mixing-length method is often preferred because those methods insure that the Reynolds shear stress is zero for zero velocity gradient (for finite $E$ or $\ell$ ). That is usually the case, although there can be exceptions for certain asymmetric flows. Some workers prefer to use the mixing length, because it seems to them easier to make an assumption for a length than for an eddy viscosity or for a Reynolds shear stress. However, it should be pointed out that the choice of Equation (3) as the definition of the mixing length is itself an assumption, since that definition is by no means the only one that might be given. For example we might have used Taylor's mixing length.16 Alternatively, a mixing length $l^{\prime}$ might be given by

$$
\overline{\mathrm{u}_{1} \mathrm{u}_{2}} \equiv-\ell^{\mathrm{q}} \mathrm{U}_{1} \partial \mathrm{u}_{1} / \partial \mathrm{x}_{2}
$$

and an assumption made for $\ell^{\prime}$.

Probably the most reasonable expression for the region away from walls is Von Karman's similarity expression. 17 That expression is most easily obtained by assuming that away from boundaries, the turbulence at a point is a function only of conditions in the vicinity of the point, in particular of the first and second velocity derivatives at the point. In this case it is equally convenient to use Reynolds stress, eddy viscosity, or mixing length (Eq. (3)), but Equation (4) is not used because its use would imply that the velocity relative to the wall is important here. If we start from the Reynolds stress and apply dimensional analysis we get

$$
\overline{u_{1} u_{2}}=\overline{u_{1} u_{2}}\left(\frac{\partial U_{1}}{\partial x_{2}}, \frac{\partial^{2} U_{1}}{\partial x_{2}^{2}}\right)=-K^{2} \frac{\left(\partial U_{1} / \partial x_{2}\right)^{4}}{\left(\partial^{2} U_{1} / \partial x_{2}^{2}\right)^{2}}
$$


where $K$ is the Karman constant. If we had started from the eddy viscosity (Eq. (2)), we would have obtalned, by dimensional analysis,

$$
\varepsilon=\varepsilon\left(\frac{\partial \mathrm{U}_{1}}{\partial \mathrm{x}_{2}}, \frac{\partial^{2} \mathrm{U}_{1}}{\partial \mathrm{x}_{2}^{2}}\right)=K^{2} \frac{\left(\partial \mathrm{U}_{1} / \partial \mathrm{x}_{2}\right)^{3}}{\left(\partial^{2} \mathrm{U}_{1} / \partial \mathrm{x}_{2}^{2}\right)^{2}}
$$

Finally, starting from the mixing length (Eq. (3)) would give

$$
\ell=\ell\left(\frac{\partial \mathrm{U}_{1}}{\partial \mathrm{x}_{2}}, \frac{\partial^{2} \mathrm{U}_{1}}{\partial \mathrm{x}_{2}^{2}}\right)=\mathrm{K} \frac{\partial \mathrm{U}_{1} / \partial \mathrm{x}_{2}}{\partial^{2} \mathrm{U}_{1} / \partial \mathrm{x}_{2}^{2}}
$$

Clearly, all three of these starting points give the same result for $\overline{u_{1} u_{2}}$.

Von Karman's hypothesis is applicable in the region away from a wall. Close to a wall we assume that the eddy viscosity, or the mixing length given by Equation (4), is a function only of quantities measured relative to a wa11, $U_{1}$ and $x_{2}$, and of $v .17$ The simplest assumption consistent with dimensional analysis and the requirement that the effect of $v$ should become small for large $x_{2}$ is then

$$
\varepsilon=E\left(U_{1}, x_{2}, \nu\right)=n^{2} U_{1} x_{2}\left(1-e^{-n^{2} U_{1} x_{2} / \nu}\right)
$$

where $n$ is an experimental constant ( $n=0.124)$. If we had started from Equation (4), rather than from Equation (2), we would have obtained the equivalent expression

$$
\ell^{v}=\ell^{\prime}\left(U_{1}, x_{2}, \nu\right)=n^{2} x_{2}\left(1-e^{-n^{2} U_{1} x_{2} / v}\right)
$$

There is a third region, the so-called wake region, where $\varepsilon$ tends to be approximately constant. That is important in free turbulent flows, but it also occurs near the outer edge of a boundary layer and near the center of a pipe. However, we can of ten neglect it in the latter two cases, particularly if we use 0.36 rather than 0.4 for the experimental constant $K$. Equations (6) and (8) give results for flow and heat (or mass) transfer in tubes and boundary layers which are in good agreement with experiment (Figs. 17 and 18).17,18

The closure assumptions used here are by no means the only ones that can be made. For instance Prandt 1 has assumed that $\ell=\mathrm{Kx}_{2}$, where $\mathrm{x}_{2}$ is the distance from a wa11, in Equation ( 3 ). 16 Van Driest 19 has modified that assumption by introducing a damping factor: 


$$
\begin{gathered}
\text { R. G. Delssler } \\
\ell=\mathrm{Kx}_{2}\left(1-\mathrm{e}^{-\mathrm{x}_{2} \sqrt{\tau_{\mathrm{w}} / \rho} / A}\right)
\end{gathered}
$$

where A is another experimental constant. Equation (10) appears to be reasonably applicable to the regions both close to and away from a wall and so is sometimes considered to be more convenient to use than Equations (6) and (8). However, there is basically no reason why one equation should apply to both regions. In fact, since the turbulence mechanism close to a wall differs from that away from a wall, two equations might be considered more reasonable.

This discussion has attempted to show how turbulence solutions can be obtained by introducing simple closures into the turbulence equations. It is hoped that it has also provided some insight into the processes occurring in turbulence.

\section{REFERENCES}

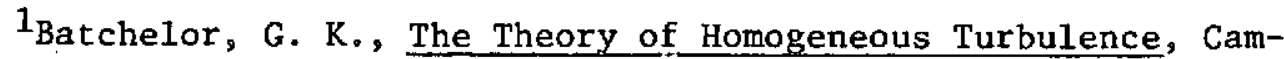
bridge University Press, New'York, 1953.

2Heisenberg, W., "Zur Statistischen Theorie der Turbulenz," Zeit. f. Phys., Vol. 124, 1948, pp. 628-657.

3ovasznay, L. S. G., "Spectrum of Locally Isotropic Turbu1ence," J. Aeron. Sci., Vo1. 15, no. 12, Dec. 1948, pp. 745753.

${ }^{4}$ Kraichnan, R. H., "Lagrangian-History Closure Approximation for Turbulence," Phys. Flulds, Vol. 8, No. 4, Apr. 1965, pp. 575-598.

${ }^{5}$ Deissler, R. G., "On the Decay of Homogeneous Turbulence Before the Final Period," Phys. Fluids, Vol. 1, No. 2, Mar.-Apr. 1958, pp. 111-121.

${ }^{6}$ Delssler, R. G., "A Theory of Decaying Homogeneous Turbulence," Phys. Fluids, Vol. 3, No. 2, Mar.-Apr.1960, pp. 176187.

${ }^{7}$ Deissler, R. G., "Decay of Homogeneous Turbulence from a Given Initial State," Phys. Fluids, Vol. 14, No. 8, Aug. 1971, pp. 1629-1638.

${ }^{8}$ Deissler, R. G., "Further Comparison of Theory and Experiment for Decay of Homogeneous Turbulence," Phys. Fluids, Vo1. 15, No. 7, July 1972, pp. 1353-1355. 
9 Deissler, R. G., "Remarks on the Decay of Homogeneous Turbulence from a Given State," Phys. Fluids, Vo1. 17, No. 3, Mar. 1974, pp. 652-653.

10 Deissler, R. G., "Effects of Inhomogeneity and of Shear Flow in Weak Turbulent Fields," Phys. Fluids, Vol. 4, No. 10, Oct. 1961, pp. 1187-1198.

11 Deissler, R. G., "Comparison of Theory and Experiment for Homogeneous Turbulence with Shear," (Submitted for Publication).

12Deissler, R. G., "Growth of Turbulence in the Presence of Shear," Phys. Fluids, Vol. 15, No. 11, Nov. 1972, pp. 19181920 .

13Deissler, R. G., "Problem of Steady-State Shear-Flow Turbulence," Phys. Fluids, Vol. 8, No. 3, Mar. 1965, pp. 391-398.

${ }^{14}$ Deissler, R. G., "Evolution of a Moderately Short Turbulent Boundary Layer in a Severe Pressure Gradịent," J. Fluid Mech., Vol. 64, Pt. 4, 1974, Pp. 763-774.

15 Deissler, R. G., "Evolution of the Heat Transfer and Flow in Moderately Short Turbulent Boundary Layers in Severe Pressure Gradients," Int. J. Heat Mass Transfer, Vo1. 17, 1974, pp. 1079-1085.

16 Hinze, J. 0., Turbulence, McGraw-Hill Book Company, Inc., New York, 1959.

17 Deissler, R. G., "Turbulent Heat Transfer and Friction in Smooth Passages," Turbulent Flows and Heat Transfer, edited by C. C. Lin, Princeton University Press, Princeton, New Jersey, 1959, pp. 288-313.

${ }^{18}$ Deissler, R. G., and Loeffler, A. L., "Analysis of Turbulent Flow and Heat Transfer on a Flat Plate at High Mach Numbers With Variable Fluid Properties," National Aeronautics and Space Administration Technical Report, R-17, 1959, Washington, D. C.

${ }^{19}$ Van Driest, E. R." "On Turbulent Flow Near a Wa11," J. Aero. Sc1., Vo1. 23, 1956, pp. 1007-1011. 


$$
\begin{gathered}
\frac{\partial u_{i}}{\partial t}+\frac{\partial\left(u_{i} u_{k}\right)}{\partial x_{k}}=-\frac{1}{\rho} \frac{\partial p}{\partial x_{j}}+v \frac{\partial^{2} u_{i}}{\partial x_{k} \partial x_{k}} \| u_{j}^{\prime} \\
\frac{\partial u_{j}^{\prime}}{\partial t}+\frac{\partial\left(u_{j}^{\prime} u_{k}^{\prime}\right)}{\partial x_{k}^{\prime}}=-\frac{1}{p} \frac{\partial p^{\prime}}{\partial x_{j}^{\prime}}+v \frac{\partial^{2} u_{j}^{\prime}}{\partial x_{k}^{\prime} \partial x_{k}^{\prime}} \| u_{i}^{\vec{r}} \\
\frac{\partial \overline{u_{i} u_{j}^{\prime}}}{\partial t}+\frac{\partial}{\partial r_{k}}\left(\overline{u_{i} u_{j}^{\prime} u_{k}^{\prime}}-\overline{u_{i} u_{j}^{\prime} u_{k}}\right)=-\frac{1}{\rho}\left(\frac{\partial p^{\prime} u_{i}}{\partial r_{j}}-\frac{\partial \overrightarrow{p_{j}^{\prime}}}{\partial r_{i}}\right)+2 v \frac{\partial^{2} \overline{u_{i} u_{j}^{\prime}}}{\partial r_{k} \partial r_{k}}
\end{gathered}
$$

Fig. 1 Jwo-point correlation equation.

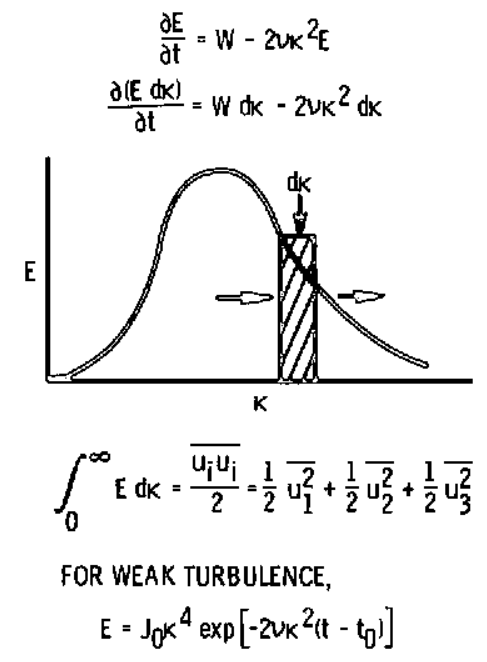

Fig. 2 Spectral equations and energy spectrum. 


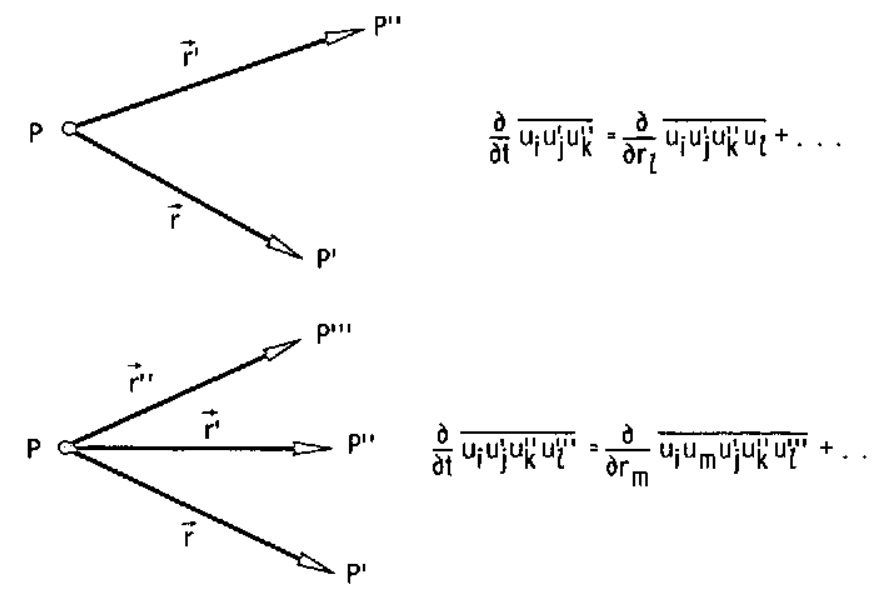

Fig. 3 Three and four point correlation equations.

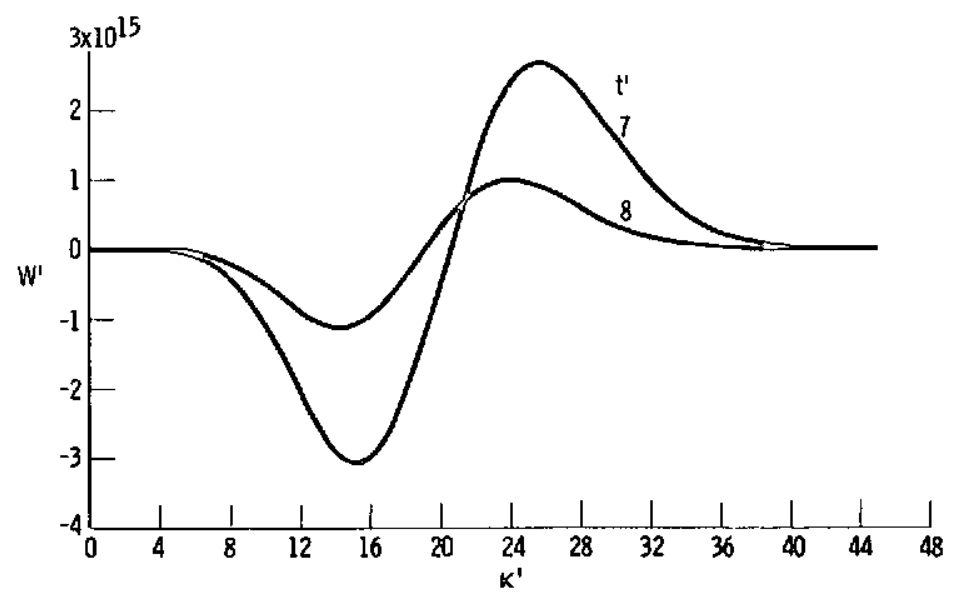

Fig. 4 Dimensionless energy transfer spectra. $W^{\prime}=\int_{0}^{5} w /\left(v^{10_{\beta_{0}}}\right)$, $k^{\prime}=J_{0}^{1 / 3} k / \nu^{2 / 3}, t^{\prime}=v^{7 / 3}\left(\mathrm{t}-\xi_{0}\right) / j_{0}^{2 / 3} \times 10^{3} . J_{0}$ and $\beta_{0}$ are constants of the initial conditions.






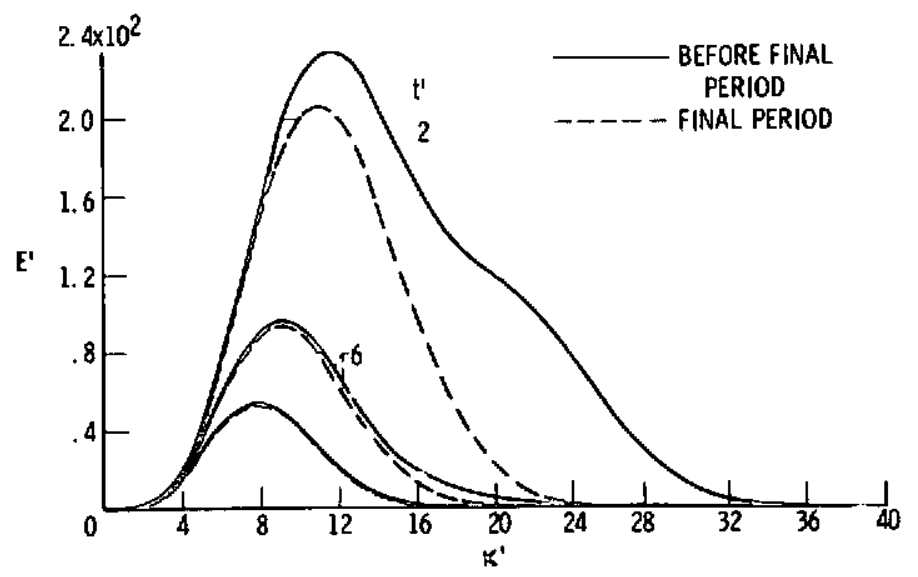

Fig. 5 Comparison of dimensionless spectra pefore fjnal period with those for final period of decay. $E^{\prime}=J_{0}^{1 / 3} \mathrm{E} / \nu^{8 / 3}$ isee Fig. 4 for definitions of other quantities).

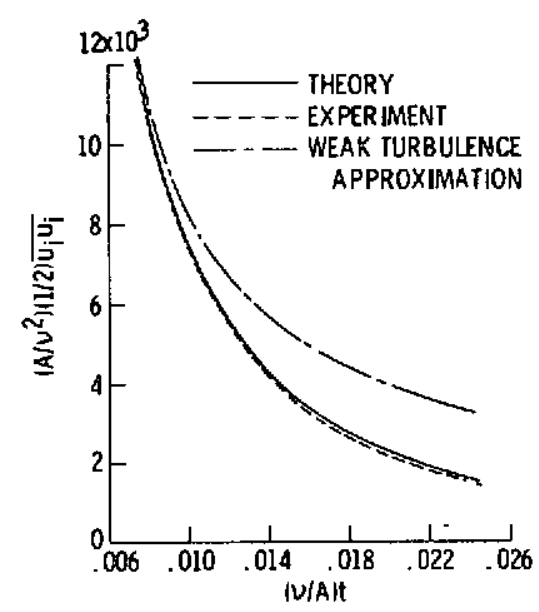

Fig. 6 Comparison of theory and experiment lor decay of turbulent energy. 


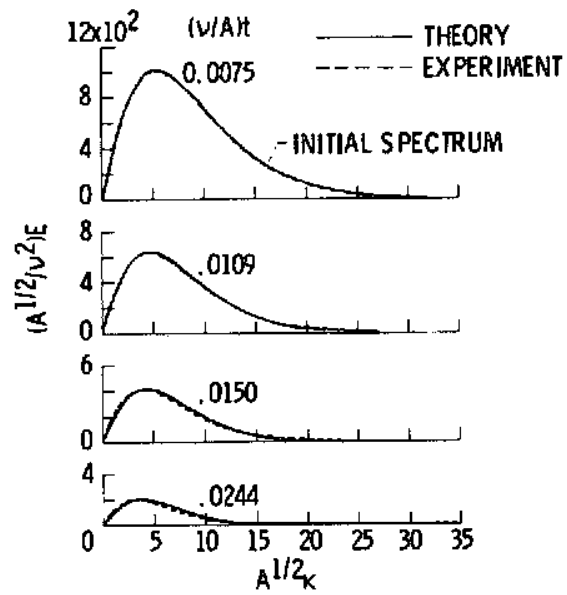

Fig. 7 Comparison of theory and experiment for decay of threedimensional turbutent-energy spectra.

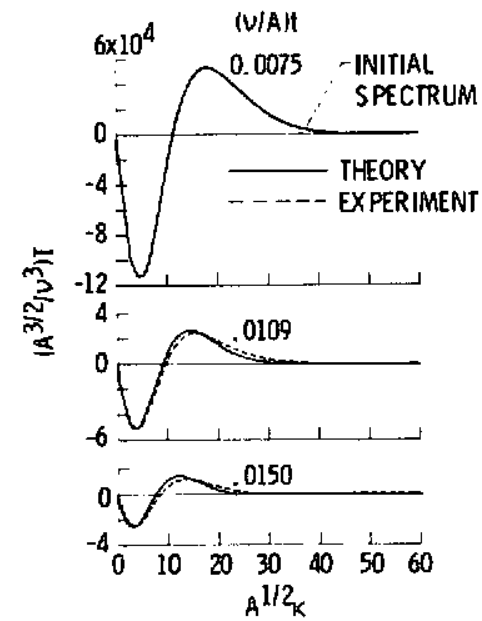

Fig. 8 Comparison of theory and experiment for decay of energy-transfer spectra. 


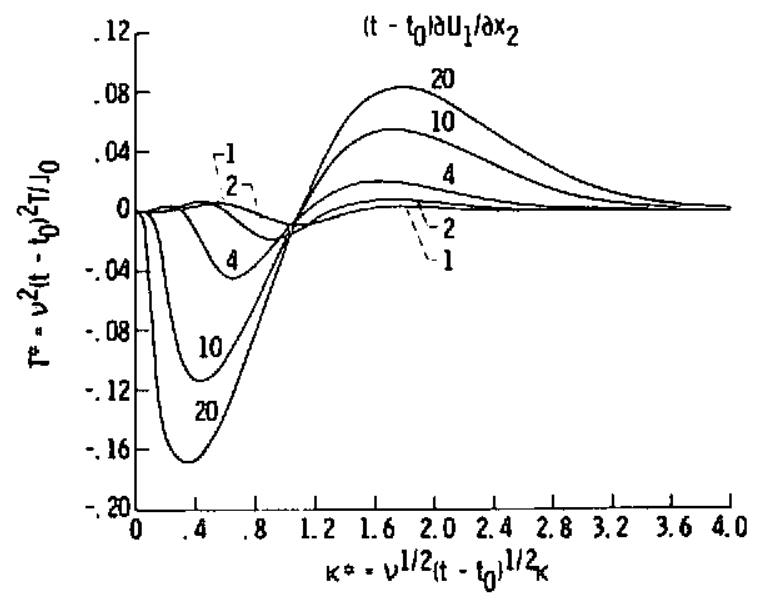

Fig. 9 Spectra of transfer term due to mean velocity gradient.

ENERGY TRANSFER

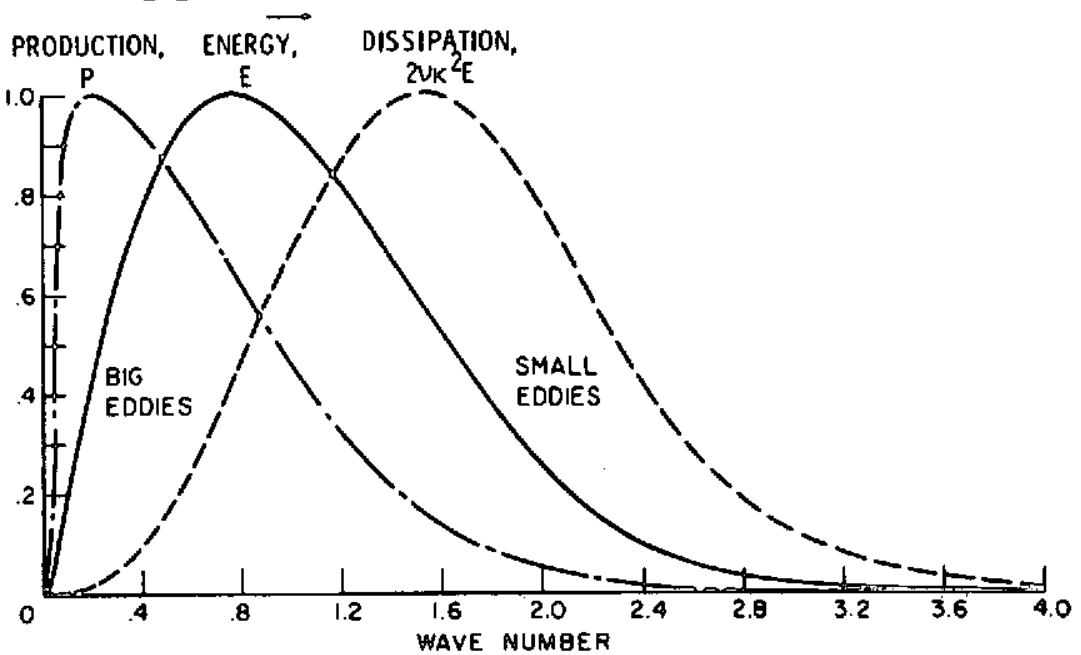

Fig. 10 Production, energy-containing, and dissipation regions. 


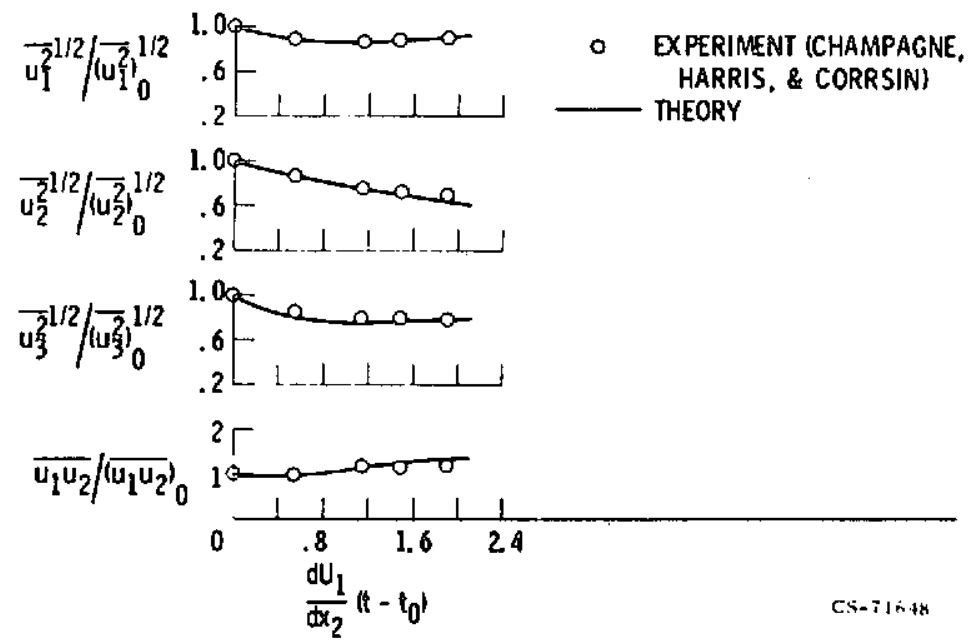

Fig. 11 Comparison of theory and experiment for evolution of one-point turbulence components with uniform shear.
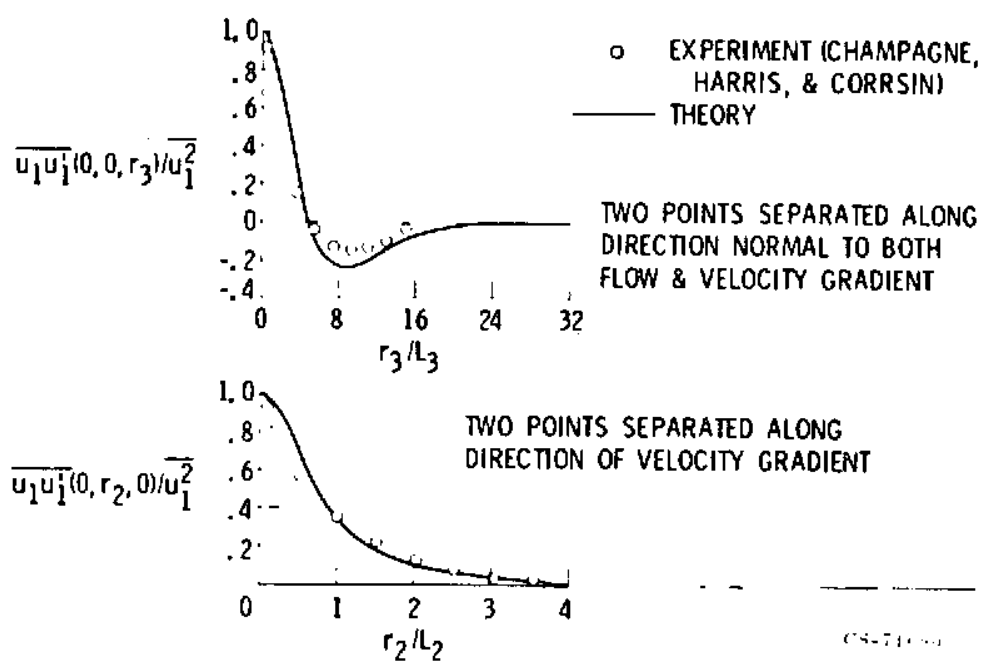

Fig. 12 Comparison of theory and experiment lor two-point velocity correlations. $\left.\quad \mathrm{du}_{1} / \mathrm{dx} \mathrm{x}_{2}\right)\left(\mathrm{t}-\mathrm{t}_{0}\right)=2.27$. 


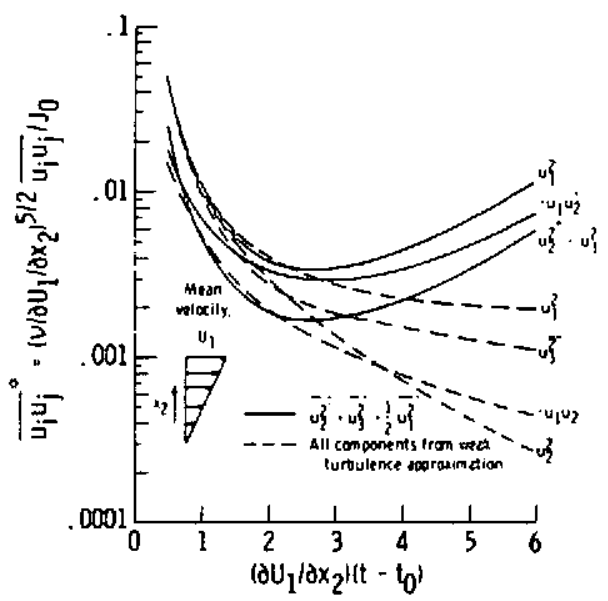

Fig. 13 Growth due to shear of weak homcgeneous turbulence when $\overline{u_{2}^{2}}=\overline{u_{3}^{2}}=\{1 / 2) u_{1}^{2}$ and comparison with case where components are all calculated from weak turbu lence approximation.

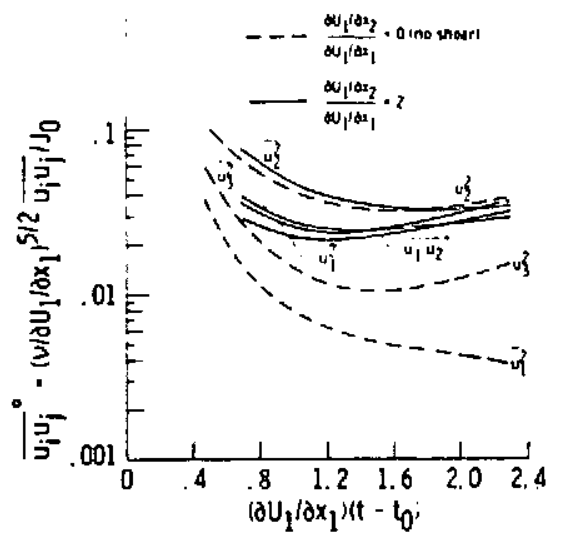

Fig. 14 Growth due to shear of weak, locally homogeneous turbulence with normal strain. 


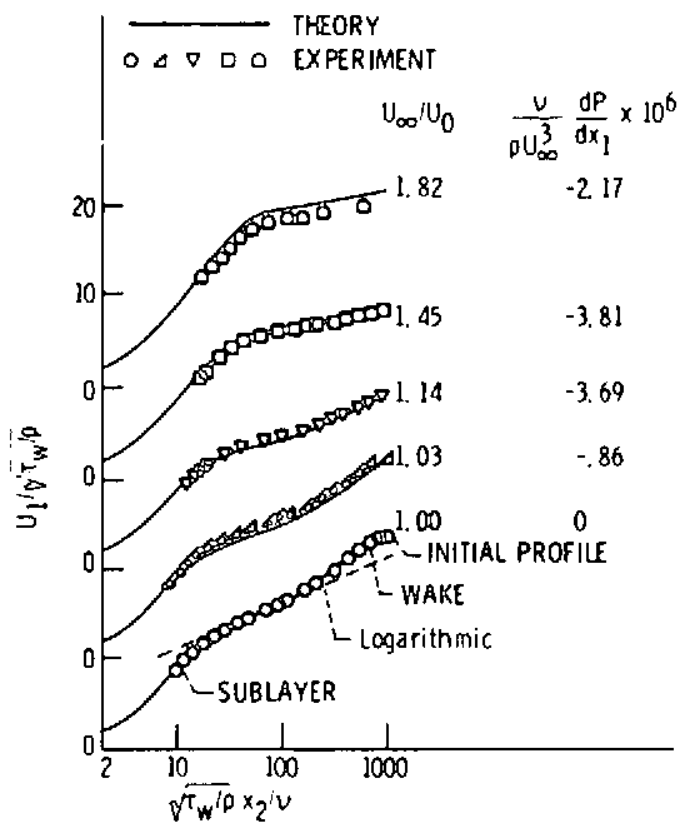

Fig. 15 Semilogarithmic law of the wall plot of theoretical velocity profiles for severe favorable pressure gradients and comparison with experiment of Patel and Head (1968). INote shifted vertical scales. !
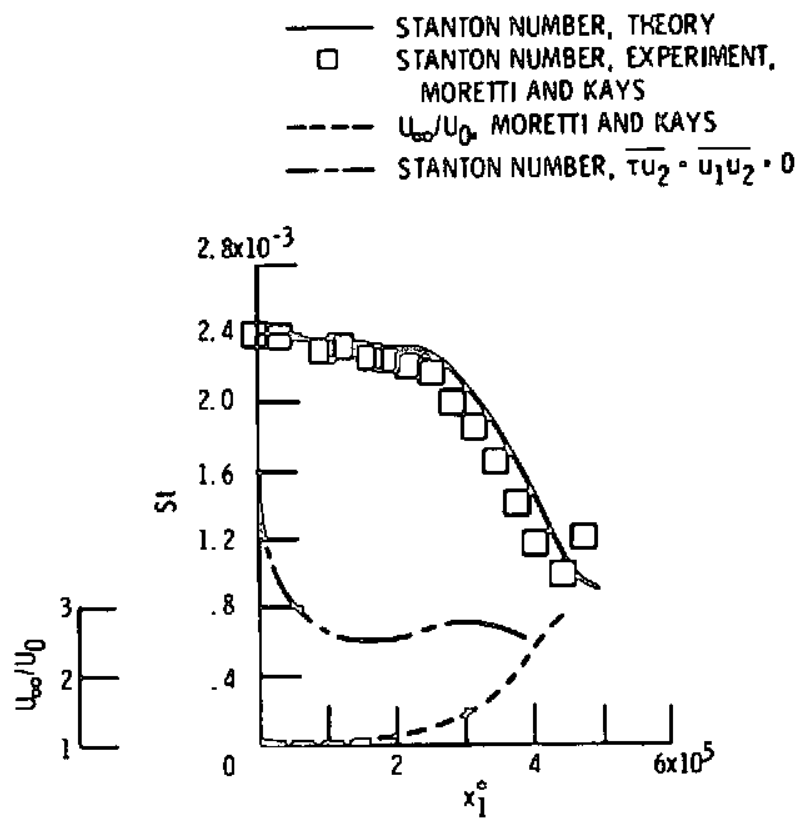

Fig. 16 Comparison of theory and experiment for evolution of Stanton number in moderately short highly accelerated turbulent boundary layers. 


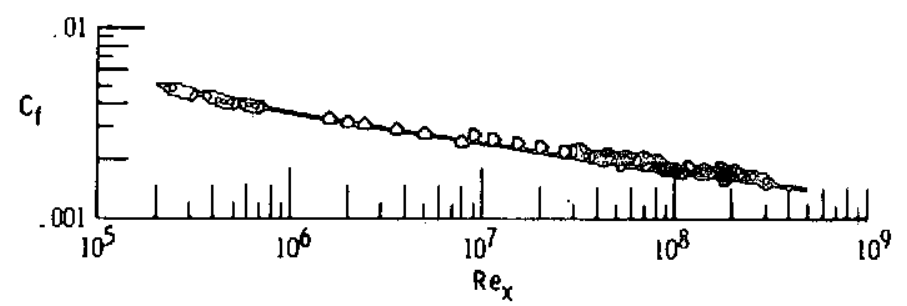

Fig. 17 Comparison of theory and experiment for flat-plate skiniriction coefficient for low speed flows.

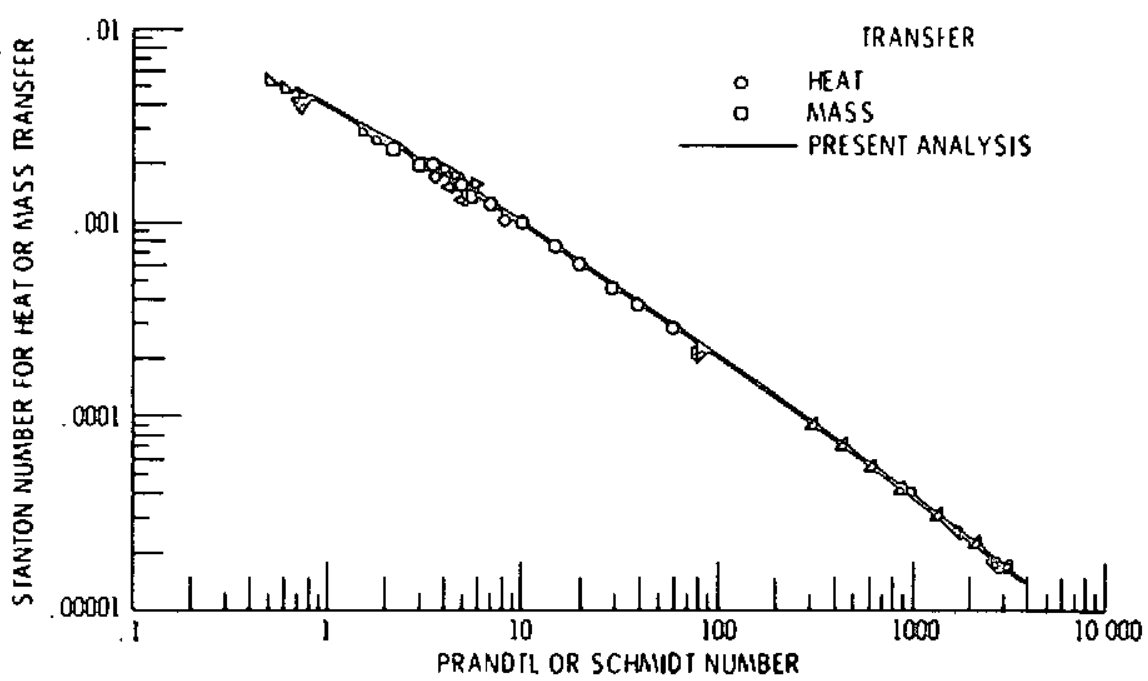

Fig. 18 Comparison of theory and experiment for fully developed heat and mass tramsfer. 